\title{
WS03 A05
}

\section{Pore-scale Processes in Amott Spontaneous Imbibition Tests}

M. Rücker* (Imperial College London), W.B. Bartels (Utrecht University), M.A. Boone (XRE), T. Bultreys (Ghent University / Imperial College London), H. Mahani (Shell Global Solutions International B.V.), S. Berg (Shell Global Solutions International B.V.)

\section{Summary}

We observed the redistribution of the oil phase in the pore space of the rock in real-time in water-wet and mixed-wet (by ageing in crude oil) carbonate samples. During the imbibition of the water phase both, pore filling events with connection to the surrounding brine as well as snap-off events connected through water films only were detected. The distribution of the oil in different pore sizes as well as the different event types help to identify the wettability state of the system and understand how pore scale processes lead to the oil production at the larger scale. 


\section{Introduction}

Amott tests are well-established macroscopic spontaneous imbibition tests to investigate wettability of oil-saturated rock plugs. In this test, the cumulative volume of produced oil versus time is used as a qualitative indicator for the macroscopic wettability of the sample (Amott, 1956). However, as wettability finds its origin on smaller length scales (Hirasaki, 1991), pore-scale processes and the pore-scale fluid distribution may give better insights into the wettability of an oil/brine/rock-system. This study, we have used X-ray micro-CT imaging at two different spatio-temporal scales, aimed at linking the macroscopically observed oil production to underlying pore-scale processes.

\section{Methods}

We used novel core holders to visualize the pore-scale fluid distribution during Amott tests. In order to verify consistency across length scales, we performed experiments on two different sample sizes of a carbonate rock (Ketton). One core holder was designed for mini-plugs (diameter: $4 \mathrm{~mm}$, length: 20 $\mathrm{mm}$ ) to capture dynamic processes during spontaneous imbibition by fast imaging (15 s/scan, voxel size: $13 \mu \mathrm{m}$ ) with the EMCT scanner (Bultreys et al., 2016; Dierick et al., 2014; www.ugct.ugent.be) from the Centre for X-ray Tomography of the Ghent University (UGCT). The other core holder was used for standard-size SCAL plugs (diameter: $3.8 \mathrm{~cm}$, length: $5 \mathrm{~cm}$ ) imaged with HECTOR (Higher Energy CT Optimized for Research, UGCT) micro-CT scanner (100 min/scan, voxel-size: $13 \mu \mathrm{m})$. The samples were saturated with brine and desaturated with decane (water-wet) or crude (mixed-wet by aging) in advance.

\section{Conclusions}

Two event types are dominant during spontaneous imbibition: pore filling events with connection to the surrounding brine and snap-off events connected through water films only. The distribution of the oil in different pore sizes as well as different event types help to identify the wettability state of the system and understand how pore-scale processes lead to the oil production at the larger scale.

\section{References}

Amott, E. [1959] Observations Relating to the Wettability of Porous Rock. Society of Petroleum Engineers.

Hirasaki, G. J. [1991]. Wettability: Fundamentals and Surface Forces. Society of Petroleum Engineers.

Bultreys, T., Boone, M.A., Boone, M.N., De Schryver, T., Masschaele, B., Van Hoorebeke, L., Cnudde, V., [2016] Fast laboratory-based micro-computed tomography for pore-scale research: Illustrative experiments and perspectives on the future. Advances in Water Resources 95:341-351.

Dierick, M., Van Loo, D., Masschaele, B., Van den Bulcke, J., Van Acker, J., Cnudde, V., Van Hoorebeke, L., [2014] Recent micro-CT scanner developments at UGCT. Nuclear Instruments and Methods in Physics Research Section B: Beam Interactions with Materials and Atoms 324:35-40. 